\title{
Decreased glutamine synthetase, increased citrulline-nitric oxide cycle activities, and oxidative stress in different regions of brain in epilepsy rat model
}

\author{
Mummedy Swamy • Wan Roslina Wan Yusof • \\ K. N. S. Sirajudeen • Zulkarnain Mustapha • \\ Chandran Govindasamy
}

Received: 22 April 2010 / Accepted: 4 October 2010 /Published online: 20 October 2010

(C) University of Navarra 2010

\begin{abstract}
To understand their role in epilepsy, the nitric oxide synthetase (NOS), argininosuccinate synthetase (AS), argininosuccinate lyase (AL), glutamine synthetase (GS), and arginase activities, along with the concentration of nitrate/nitrite (NOx), thiobarbituric acid reactive substances (TBARS), and total antioxidant status (TAS), were estimated in different regions of brain in rats subjected to experimental epilepsy induced by subcutaneous administration of kainic acid (KA). The short-term (acute) group animals were killed after $2 \mathrm{~h}$ and the long term (chronic) group animals were killed after 5 days of single injection of KA $(15 \mathrm{mg} / \mathrm{kg}$ body weight). After decapitation of rats, the brain regions were separated and in their homogenates, the concentration of NOx, TBARS and TAS and the activities of NOS, AS, AL, arginase and glutamine synthetase were assayed by colorimetric methods. The results of the study demonstrated the increased activity of NOS and formation of NO in acute and chronic groups epilepsy. The activities of AS and AL were increased and indicate the effective recycling of citrulline to
\end{abstract}

M. Swamy $(\bowtie) \cdot$ W. R. W. Yusof $\cdot$ K. N. S. Sirajudeen •

Z. Mustapha $\cdot$ C. Govindasamy

Department of Chemical Pathology, School of Medical

Sciences, Health Campus, Universiti Sains Malaysia,

Kubang Kerian 16150, Kelantan, Malaysia

e-mail: mswamy@kb.usm.my

M. Swamy

e-mail: mummedys@yahoo.co.in arginine. The activity of glutamine synthetase was decreased in acute and chronic groups of epilepsy compared to control group and indicate the modulation of its activity by NO in epilepsy. The activity of arginase was not changed in acute group; however it was decreased in chronic group and may favor increased production of NO in this condition. The concentration TBARS were increased and TAS decreased in acute and chronic groups of epilepsy and supports the oxidative stress in epilepsy.

Keywords Nitric oxide - Citrulline - NO cycle enzymes · Glutamine synthetase $\cdot$ Thiobarbituricacid reactive substances $\cdot$ Total antioxidant status $\cdot$ Epilepsy

\section{Introduction}

Neuronal excitation involving the excitatory glutamate receptors is recognized as an important underlying mechanism in neurodegenerative disorders [11]. Glutamate and related excitatory amino acids are considered major neurotransmitters in the central nervous system [10]. In the CNS, the conversion of glutamate to glutamine by glutamine synthetase (GS), that takes place within the astrocytes, represents a key mechanism in the regulation of excitatory neurotransmission under normal conditions as well as in injured brain [41]. Kainic acid (KA) is a potent CNS excitotoxin, producing acute and sub-acute epileptic- 